EPJ Web of Conferences 59, 01010 (2013)

DOI: $10.1051 /$ epjconf/20135901010

(C) Owned by the authors, published by EDP Sciences, 2013

\title{
Table-top solar flares produced with laser driven magnetic reconnections
}

\author{
J.Y. Zhong ${ }^{1}$, Y.T. Li ${ }^{2}$, X.G. Wang ${ }^{3}$, J.Q. Wang ${ }^{3}$, Q.L. Dong ${ }^{2}$, X. Liu², X.X. Lin², \\ D.W. Yuan ${ }^{2}$, F. Du², S.J. Wang ${ }^{2}$, L. Zhang ${ }^{2}$, L. An ${ }^{2}$, C.J. Xiao ${ }^{3}$, H.G. Wei ${ }^{1}$, \\ K. Zhang ${ }^{1}$, F.L. Wang ${ }^{1}$, S.E. Jiang ${ }^{4}$, Y.K. Ding ${ }^{4}$, Z.R. Cao ${ }^{4}$, Z. Yuan ${ }^{4}$, \\ H.Y. Zhang ${ }^{4}$, Z.W. Yang ${ }^{4}$, J.Q. Zhu ${ }^{5}$, X.T. He ${ }^{6,7,8}$, H.B. Cai ${ }^{7}$, G. Zhao ${ }^{1}$ \\ and J. Zhang ${ }^{9}$
}

${ }^{1}$ Key Laboratory of Optical Astronomy, National Astronomical Observatories, Chinese Academy of Sciences, Beijing100012, China

${ }^{2}$ Beijing National Laboratory for Condensed Matter Physics, Institute of Physics, Chinese Academy of Sciences, Beijing 100190, China

${ }^{3}$ State Key Laboratory of Nuclear Physics \& Technology, School of Physics,

Peking University, Beijing 100871, China

${ }^{4}$ Research Center for Laser Fusion, China Academy of Engineering Physics, Mianyang 621900, China

${ }^{5}$ National Laboratory on High Power Lasers and Physics, Shanghai 201800, China

${ }^{6}$ Center for Applied Physics and Technology, Peking University, Beijing 100871, China

${ }^{7}$ Institute for Fusion and simulation, Zhejiang University, Hangzhou 310027, China

8 Institute of Applied Physics and Computational Mathematics, Beijing 100094, China

${ }^{9}$ Departmem of Physics, Shanghai Jiao Tong University, Shanghai 200240, China

\begin{abstract}
The American Nuclear Society (ANS) has presented the prestigious Edward Teller award to Dr. Bruce A. Remington during the 2011 IFSA conference due to his "pioneering scientific work in the fields of inertial confinement fusion (ICF), and especially developing an international effort in high energy density laboratory astrophysics" $[1,2]$. This is a great acknowledgement to the subject of high energy density laboratory astrophysics. In this context, we report here one experiment conducted to model solar flares in the laboratory with intense lasers [3]. The mega-gauss -scale magnetic fields produced by laser produced plasmas can be used to make magnetic reconnection topology. We have produced one table-top solar flare in our laboratory experiment with the same geometric setup as associated with solar flares.
\end{abstract}

\section{INTRODUCTION}

Traditional astrophysical phenomena are pursued with observations and theoretical modelling. The recent advances in observations and modelling provide a solid ground for modern astrophysics. The growing field of high-energy density laboratory astrophysics (HEDLA) seeks to understand the physics involved in fusion applications and astrophysical phenomena in laboratory astrophysics. Contemporary research in laboratory astrophysics utilizes powerful, megajoule class lasers such as at the National Ignition Facility (NIF) and other laser fusion facilities like Omega lasers and GEKKO lasers in Japan. In China, Shenguang-II laser facility is used for the study of HEDLA phenomena. These high energy density laser facilities provide unique opportunities to study fundamental material properties such as

This is an Open Access article distributed under the terms of the Creative Commons Attribution License 2.0, which permits unrestricted use, distribution, and reproduction in any medium, provided the original work is properly cited. 


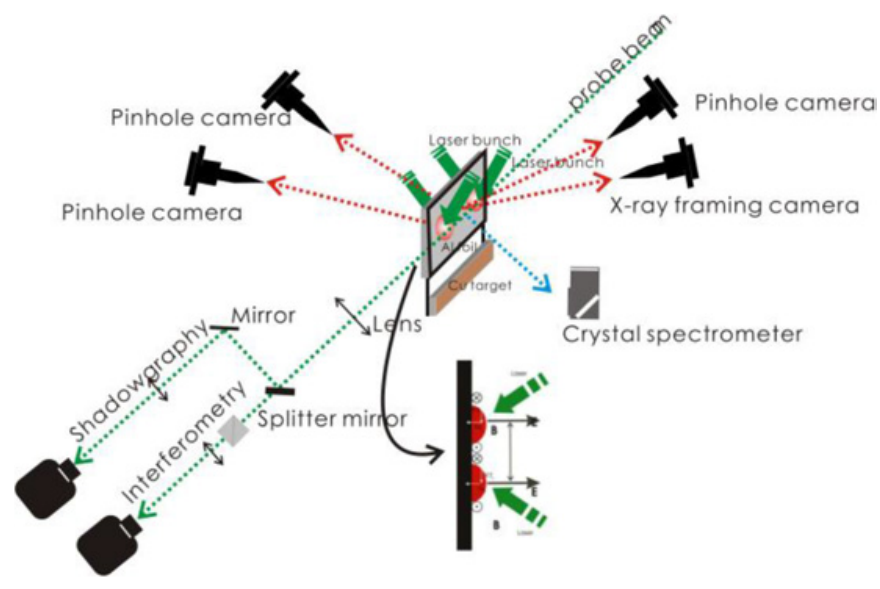

Figure 1. Schematic view of the experimental setup for laser-driven magnetic reconnection. Two laser beams irradiate the target which results in two plasma bubbles. The magnetic fields are anti-parallel between the plasma bubbles. Magnetic reconnection occurs when the two plasma bubbles encounter.

opacity [4], equations of state [5], photoionized plasmas [6,7], as well as the similarity of physics involved in certain hydrodynamic phenomena of jets [8] and shocks [9] when a scaling law between astrophysical and laboratory plasma systems can be applied. Consequently, the main focus of laboratory astrophysics is to simulate fundamental nature of astrophysical plasma physics processes in a laboratory environment so that certain astrophysical phenomenon can be studied in a well controlled manner $[1,2,10]$.

Magnetic reconnection (MR) is a fundamental physical process in a plasma which facilitates the conversion of magnetic energy into kinetic and thermal energies through acceleration and/or heating of particles [11, 12], and is believed to dominate among different astrophysical phenomena from solar flares [13, 14], star formations [15], other astrophysical events [16], laser-produced plasma jets [17-19], tokamak sawteeth [20] to disruptions in laboratories. Considerably large scale differences between astrophysical and laboratory plasmas hinder the direct measurements of magnetic reconnection events in laboratory. However, with proper scaling law [21] between different magnetohydrodynamic (MHD) systems, certain astrophysical phenomena can possibly be envisioned in laboratory such as a solar wind driven magnetosphere [22].

In this article we present a well-scaled laboratory experiment producing a table-top solar flare in which the MR topology is constructed with spontaneously generated magnetic field in high power laser-plasma interactions. This non-equilibrium laser driven magnetic reconnection is quite similar with the real astrophysical MR phenomena, which might open a new direction in the field of laboratory astrophysics.

\section{EXPERIMENTAL SETUP}

The experiment is performed on the Shenguang (SG) II laser facility in China, which can deliver a total energy of $2.0 \mathrm{~kJ}$ in a nanosecond square pulse. The eight SG-II laser beams $\left(\lambda_{\mathrm{L}}=0.351 \mu \mathrm{m}\right)$ are divided into four bunches and each bunch contains two laser beams. The geometric configuration as shown in Fig. 1 is designed to setup one standard laser driven magnetic reconnection topology. The two synchronized laser bunches separated by $200-800 \mu \mathrm{m}$ are then focused on one side of the Al foil, while the other two bunches shine the other side of the target symmetrically and simultaneously. Each bunch is focused to a focal spot diameter of 50-100 $\mu \mathrm{m}$ in full width at half maximum (FWHM) generating an incident laser intensity of $\sim 5 \times 10^{15} \mathrm{~W} / \mathrm{cm}^{2}$. A copper target is placed at $250 \mu \mathrm{m}$ away from the 
Table 1. Typical parameters in solar flare and laser plasmas.

\begin{tabular}{|c|c|c|c|}
\hline Parameters & Solar flare [30, 31] & Laser plasmas & Scaled flare plasmas \\
\hline Length $(\mathrm{cm})$ & $10^{9}-10^{10}$ & $10^{-1}$ & $10^{-2}-10^{-1}$ \\
hline Time $(\mathrm{s})$ & $100-1,000$ & $10^{-9}$ & $10^{-10}-10^{-9}$ \\
\hline Pressure $(\mathrm{Pa})$ & $0.01-10$ & $10^{7}$ & $10^{7}-10^{11}$ \\
\hline Density $(\mathrm{cm}-3)$ & $\sim 10^{10}-10^{11}$ & $\sim 10^{19}-10^{20}$ & $10^{19}-10^{21}$ \\
\hline Velocity $(\mathrm{km} \mathrm{s}-1)$ & $\sim 10-100$ & $\sim 100$ & $100-1,000$ \\
\hline Magnetic field $(\mathrm{G})$ & $\sim 10-100$ & $\sim 10^{6}$ & $10^{6}-10^{7}$ \\
\hline
\end{tabular}

$\mathrm{Al}$ foil edge. The $\mathrm{Al}$ foil size is $1,600 \mu \mathrm{m} \times 500 \mu \mathrm{m}$ and in thickness of $10-50 \mu \mathrm{m}$, while the $\mathrm{Cu}$ target is $1,600 \mu \mathrm{m} \times 250 \mu \mathrm{m}$ with the thickness of $150 \mu \mathrm{m}$. The X-ray emission is measured by three X-ray pinhole cameras in the forward, side and reverse directions to investigate the reconnection jets as well as their impact on the $\mathrm{Cu}$ target. The image is taken with $10-\mu \mathrm{m}$ pinhole filtered with $50 \mu \mathrm{m}$ of beryllium allowing all X-rays with energy above $\sim 1 \mathrm{keV}$. Most of the signal from high-energy continuum is time-integrated recorded on an X-ray film which is sensitive to X-rays in the $1-10 \mathrm{keV}$ range. A flat crystal spectrometer is set in front of the targets to record the X-ray spectra from the heating plasmas. Shadowgraphy and interferometry with a 120 ps green $\left(\lambda_{\mathrm{L}}=0.53 \mu \mathrm{m}\right)$ laser beam are also employed to investigate plasma evolution. A single-frame X-ray framing camera has been set up for imaging of X-ray emissions from laser magnetic reconnection sources. It consists of two parts: an X-ray pinhole camera using an open-ended microchannel plate (MCP) detector coupled to a CCD camera, and a high voltage short duration gate pulse of $70 \mathrm{ps}$ for the MCP. The camera uses a $20-\mu \mathrm{m}$ pinhole aperture for recording image on the MCP detector with a magnification of 3X. The evolutions of 2D X-ray images are recorded with very high-speed time resolution (about $54 \mathrm{ps).}$

\section{RESULTS AND DISCUSSIONS}

\subsection{Magnetic reconnection in solar flares and laser driven magnetic reconnection}

A solar flare is a fierce explosion in the sun's atmosphere that emits a burst of radiation. One big solar flare can affect space environment and human beings life on earth very seriously. There are many observed evidences that support the magnetic reconnection model of solar flare. In 1992, Masuda et al [23] observed the loop-top X-ray source in solar flares by using the YOHKOH satellite and proposed that two antiparallel magnetic fields were merged above an arcade of closed loops as outflows from the reconnection point which collided with high density plasmas on the loop to produce a hot X-ray region. The observations of Ultraviolet [24] and X-ray [25, 26] plasma jets ejected from the regions above the solar surface also confirm the theoretical models of MR. Table 1 list the typical solar flares parameters.

On the other side, ultra-strong magnetic fields up to mega-gauss scale can be generated via the interactions of ultra-intense lasers with plasmas. The mechanism of self-generated magnetic field in laser plasmas is different due to the laser conditions. Thermoelectric mechanism is most important with the long pulse lasers (nanosecond), where temperature and density gradients on the target surface are nonparallel. Toroidal magnetic fields have been measured successfully with different methods. The main features of self-generated magnetic fields include: (a) strong magnetic field of mega-gauss scale (b) concentrated on a hemispherical plasma bubble (c) surrounding and expanding laterally. Since the magnetic reconnection is the breaking and re-organizing of magnetic field lines which need to satisfy two requirements: firstly magnetized plasmas encounter each other and secondly the anti-parallel pointing B-fields. By considering the features of self-generated magnetic field in laser plasmas, we can construct the MR topology with two laser beams as shown in figure 1. Yates et al [27] observed the hot emission 

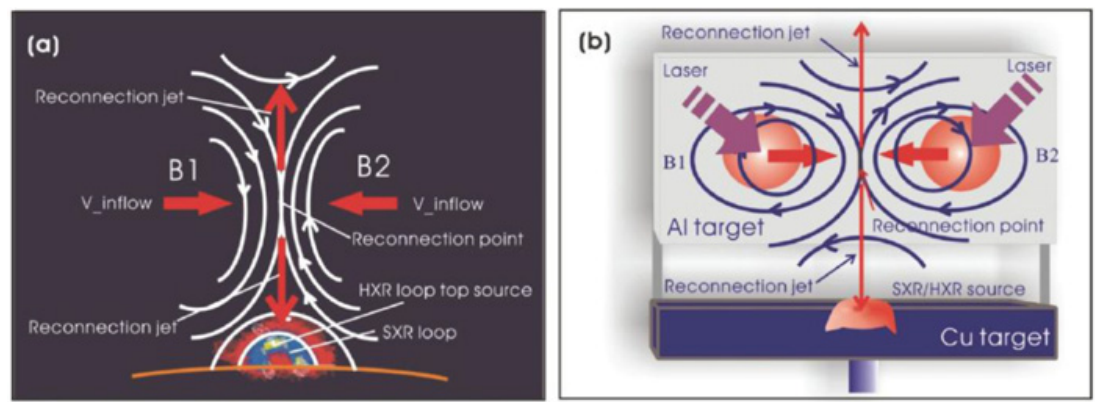

Figure 2. (a) Cartoon shows the loop-top x-ray source in solar flares: white lines show the magnetic reconnection topology and red arrows show the inflows (thick) and outflows (thin). (b) The target is setup to simulate setup (a) where the MR topology can be constructed with intense lasers on the $\mathrm{Al}$ target, while the $\mathrm{Cu}$ target is presented to simulate the solar limb.

at reconnection point by focussing two laser beams on the gold foil. Nilson et al $[17,18]$ performed a similar experiment, in which two distinctive plasma jets were observed. Li et al. [28] and Willingale et al. [29] diagnosed the laser-driven magnetic reconnection process with novel proton radiography. The typical laser plasmas parameters used in our experiment are listed in Table 1 along with solar flare parameters in column 2. When compared with the solar flare parameters, one can see that the laser plasma is short lived and is of small size as well.

\subsection{MHD scaling laws}

As mentioned in section 3.1, we demonstrate two plasma systems, solar flare and laser plasmas. Magnetic reconnection can occur in both of these systems. The MR process can reasonably be described by MHD, since the magnetic Reynolds number is very high. Ryutov et al [21] reported the scaling relations of two ideal MHD systems $\left(\operatorname{Re}_{\mathrm{M}} \gg 1\right)$. The variables of the systems remain invariant under these transformations, as $r=a r_{1}, \rho=\rho_{1}, p=c p_{1}, t=a \sqrt{\frac{b}{c}} t_{1}, v=a \sqrt{\frac{c}{b}} v_{1}, B=\sqrt{c} B_{1}$, where $\mathrm{r}$ is the characteristic length, $\rho$ is the mass density, $\mathrm{p}$ is the pressure, $\mathrm{v}$ is the velocity, $\mathrm{B}$ is the magnetic field of the systems, while a, b, c are transformation coefficients.

Brady et al [22] simulated magnetosphere-solar wind interactions in laboratory with the help of MHD scaling law. By selecting the laser parameters and target materials properly, the magnetic Reynolds number $R_{e M} \approx 0.8 \sqrt{\frac{Z+1}{Z-1}} L(\mathrm{~cm})[T(\mathrm{eV})]^{2}$ is around 4,000 (for $\mathrm{Z}=13, \mathrm{~A}=27, \mathrm{~L}=0.1 \mathrm{~cm}$, $\mathrm{T}=1 \mathrm{keV})$, which makes the MHD processes in laser plasmas and solar flares $\left(\operatorname{Re}_{\mathrm{M}} \sim 5 \times 10^{8}\right)$ comparable. The MHD comparison of solar flares and laser-produced plasmas is shown in Table 1 with the transformation coefficients $\mathrm{a}=10^{-11}, \mathrm{~b}=10^{8}$, and $\mathrm{c}=10^{10}$. The scaled parameters of solar coronal plasmas in the third column are very similar to those of laser-produced plasmas listed in the second column.

\subsection{Table-top solar flare produced with intense lasers}

In astrophysical simulations the geometry of the experimental setup should be similar to that of the astrophysical case. In our experiment, we set up the target which has similar magnetic topology with the solar flare as shown in figure 2(a). The loop-top x-ray source and reconnection jets had been reported in our previous work [3]. Here, we show the dynamic x-ray images observed with framing camera. In solar flare observations, soft x-ray images which are considered to represent magnetic field-line configuration visualize examples of global topology change of plasma configurations. In laser plasmas, we also plot the magnetic field-line over the x-ray emissions. Figure 3 shows $x$-ray framing images 

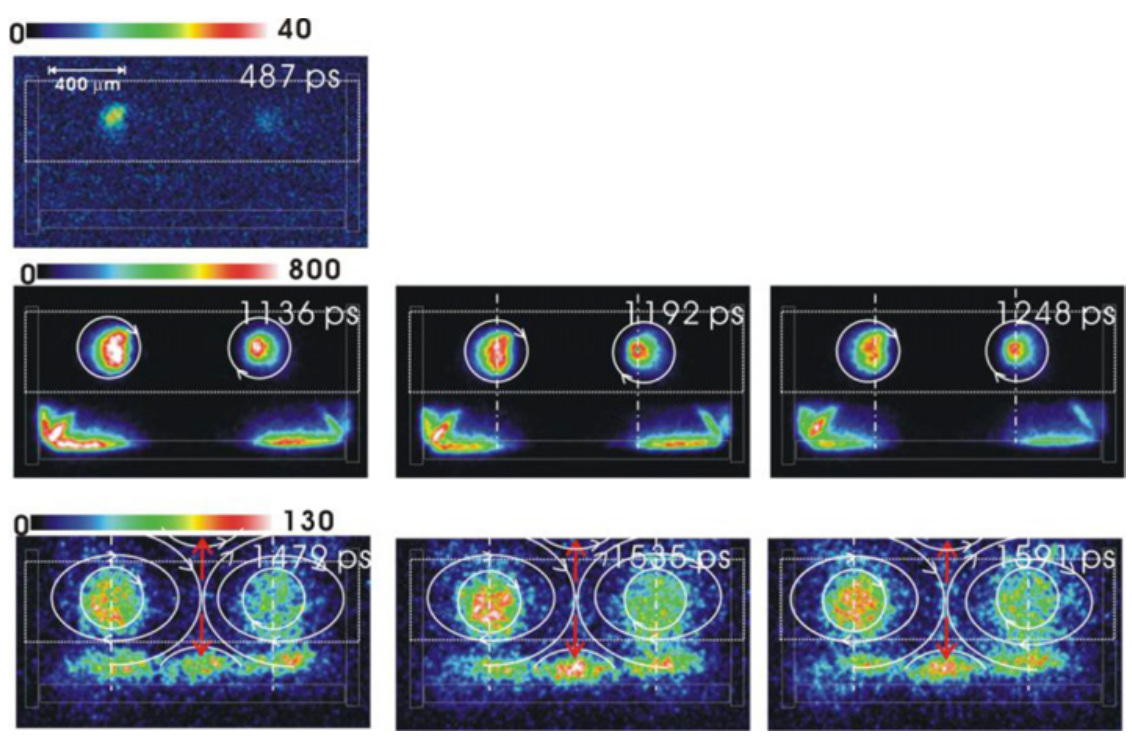

Figure 3. X-ray framing images where the two laser spots are $800 \mu \mathrm{m}$ apart. The large rectangle is $\mathrm{Al}$ target and the small rectangle is $\mathrm{Cu}$ target, refer to figure 2(b).

when the two laser spots are separated by $800 \mu \mathrm{m}$ at different time. The most distinctive features of laser driven magnetic reconnection are $\mathrm{x}$-ray emissions at encounter plane of two plasma bubbles and also outflows as shown in figure 2 (b), which will interact with $\mathrm{Cu}$ target and re-emit $\mathrm{x}$-rays at the $\mathrm{Cu}$ target. At the time of $487 \mathrm{ps}$ when the laser is still on, two weak (see scale bar) $\mathrm{x}$-ray emissions come from two focal spots. In the second row, the laser is just turned off and x-ray emission reaches its maximum peak value at focal spots. Some x-ray emissions (jet-like) are also observed at the corner of the $\mathrm{Cu}$ target, while reconnection does not occur because at that time there is no x-ray emissions at middle of two plasma bubbles of $\mathrm{Al}$ target. At later time of $1.479 \mathrm{~ns}$ (third row), in addition to x-ray emission in diffusion region from the $\mathrm{Al}$ target, three bright spots on the $\mathrm{Cu}$ target are also found. The side spots are evolution of jet-like x-ray emissions as shown in second row, while the central spot is the result of reconnection outflows interacted with $\mathrm{Cu}$ target. Several features are found similar in both systems (the present experimental plasmas and solar flare plasmas) such as reconnection jets and loop top xray source. By considering the scaling relations between these two systems we can conclude that one table-top solar flare could be produced with intense lasers.

\section{CONCLUSIONS}

Laser driven magnetic reconnection is an area of exploring a parametric space that was not previously accessible in laboratory experiment. With the application of scaling laws, we can simulate a number of astrophysical phenomena pertaining to MR, such as solar flare, accretion disk etc. In future experiments, we will continue to investigate the MR related particles acceleration. The magnetic field produced by short laser pulse is much higher than that produced with ns lasers. Using these mechanisms for magnetic field generation, we can also study the relativistic magnetic reconnection.

\section{References}

[1] Remington, B. A., Arnett, D, Drake, P \& Takabe H., Science 284,1488 (1999)

[2] Remington, B. A., Drake, P \& Ryutov, Rev. Mod. Phys. 78, 755-807 (2006) 
[3] Zhong, J. Y. et al., Nature Phys. 6, 984-987 (2010)

[4] Takabe, H. Progress of Theoretical Physics Supplement 143, 202-265 (2001)

[5] Rogers, Forest J., \& Carlos A. Iglesias., Science 263, 50-55 (1994)

[6] Celliers, P. M. et al., Phys. Plasmas 11, L41-L44 (2004)

[7] Foord, M. E. et al., Phys. Rev. Lett. 93, 055002 (2004)

[8] Fujioka, S. et al. . Nature Phys. 5, 821-825 (2009)

[9] Lebedev, S. V. et al., Astrophys. J. 564, 113-119 (2002)

[10] Foster, J. M. et al., Phys. Plasmas 9, 2251-2263 (2002)

[11] Yamada, M. Phys. Plasmas 14, 058102 (2007)

[12] Zweibel, E \& Yamada, M. Annu. Rev. Astron. Astrophys. 47, 291-332 (2009)

[13] Sweet, P. A. IAU symp. 6, ed. B. Lehnert, Cambridge Univ. Press, London, p. 123 (1958)

[14] Parker, E. N. J. Geophys. Res. 62, 509 (1957)

[15] Kulsrud, R. M., Phys. Plasmas 5, 1599 (1998)

[16] Goodman, J. \& Uzdensky, D., Astrophys. J., 688, 555-558 (2008)

[17] Nilson, P. M. et al. Phys. Rev. Lett. 97, 255001 (2006)

[18] Nilson, P. M. et al. Phys. Plasmas 15, 092701 (2008)

[19] Li, C. K. et al. Phys. Rev. Lett. 102, 205001 (2009)

[20] Taylor, J. B. 58, 741-763 (1986)

[21] Ryutov, D. D., Drake, R. P., \& Remington, B. A., Astrophys. J. 127 (Suppl.), 465-468 (2000)

[22] Brady, P., Ditmire, T., Horton, W., Mays, M. L., \& Zakharov, Y., Phys. Plasmas 16, 043112 (2009)

[23] Masuda, S. et al., Nature 371, 495-497 (1994)

[24] Innes, D. E., Inhester, B., Axford, W. I. \& Wilhelm, K., Nature 386, 811-813 (1997)

[25] Cirtain, J. W., et al., Science, 318, 1580-1582 (2007)

[26] Shibata, K., et al., Science, 318, 1591-1594 (2007)

[27] Yates, M. A., van Hulsteyn, D. B., Rutkowski, H., Kyrala, G. \& Brackbill, J. U., Phys. Rev. Lett. 49, 1702-1704 (1982)

[28] Li, C. K. et al., Phys. Rev. Lett. 99, 055001 (2007)

[29] L. Willingale et al.,Phys. Plasmas 17, 043104 (2010)

[30] Bray, R. J., Cram, L. E., Durrant, C. \& Loughhead, R. E., (Cambridge University Press, 1991)

[31] Lin, J., et al., Astrophys. J, 658, L123-L126 (2007) 